\title{
Cyto-morphological Studies of the Genus Glycyrrhiza in Iran
}

\author{
Masoud Sheidai ${ }^{1, *}$, Shokooh Iraj ${ }^{2}$, Roya Karamian ${ }^{2}$ and \\ Masoud Ranjbar ${ }^{2}$ \\ ${ }^{1}$ Shahid Beheshti University, GC, Faculty of Biological Sciences, Tehran, Iran \\ ${ }^{2}$ Biology Department, Booalisina University, Hamedan, Iran
}

Received June 18, 2008; accepted November 25, 2008

\begin{abstract}
Summary Morphological and cytogenetic analyses were performed in populations of Glycyrrhiza species growing in Iran. Clustering of the species based on morphological characters indicated distinctness of the Glycyrrhiza species studied. The present study revealed the presence of $2 n=2 x=16$ chromosome number in the species studied, reporting the chromosome number of G. triphylla for the first time. Moreover, multipolar cells, abnormal tetrads and unreduced pollen grain formation are reported in Glycyrrhiza for the first time. Clustering of the Glycyrrhiza species and populations based on morphological and cytogenetic data showed close relationship between G. glabra and G. triphylla and between G. echinata and G. aspera supporting taxonomic treatment of the genus Glycyrrhiza in Flora Iranica.
\end{abstract}

Key words Cytology, Glycyrrhiza, Morphology.

The genus Glycyrrhiza L. (family Fabaceae) is comprised of about 30 species with a sub-cosmopolitan distribution in temperate and hot temperate regions including Asia, Australia, Europe, and the Americas (Barghi and Siljak-Yakolev 1990). According to Flora Iranica (Rechinger 1984) four Glycyrrhiza species grow in Iran. The genus is best known for liquorice which is the product of G. glabra, a species native to the Mediterranean region. Russian Liquorice (G. echinata) and Chinese Liquorice (G. uralensis, "sweet grass" or "sweet herb") are also cultivated, the latter being important in traditional Chinese medicine. The liquorice is the root of G. glabra, from which a sweet flavor can be extracted.

Liquorice extract is traded both in solid and syrup form. Its active principle is glycyrrhizin, a sweetener more than 50 times as sweet as sucrose which also has pharmaceutical effects. Powdered liquorice root is an effective expectorant, and has been used for this purpose since ancient times. Modern cough syrups often include liquorice extract as an ingredient (Krausse et al. 2004).

In general a limited biosystematic or phylogenetic studies are available about the genus Glycyrrhiza throughout the world (Verma and Nadkarni 1985, Yamazaki et al. 1994, Barghi and SiljakYakolev 1990) while similar studies are totally lacking from Iran. Therefore the present study considers the biosystematic study of the genus Glycyrrhiza in Iran for the first time.

Materials and methods

\section{Morphometry}

The species studied are: 1- G. aspera Pall., 2- G. glabra L. var. Glabra., 3- G. glabra L. var. Glandulifera (Waldst and Kit.) Boiss., 4- G. echinata L. and 5- G. triphylla F. et M. Details of the localities and the voucher numbers may be asked from the first author on request. For morphometric analyses, minimum 5 and maximum 10 plants were studied from each population. Voucher spec-

\footnotetext{
*Corresponding author, e-mail: msheidai@sbu.ac.ir, msheidai@yahoo.com
} 


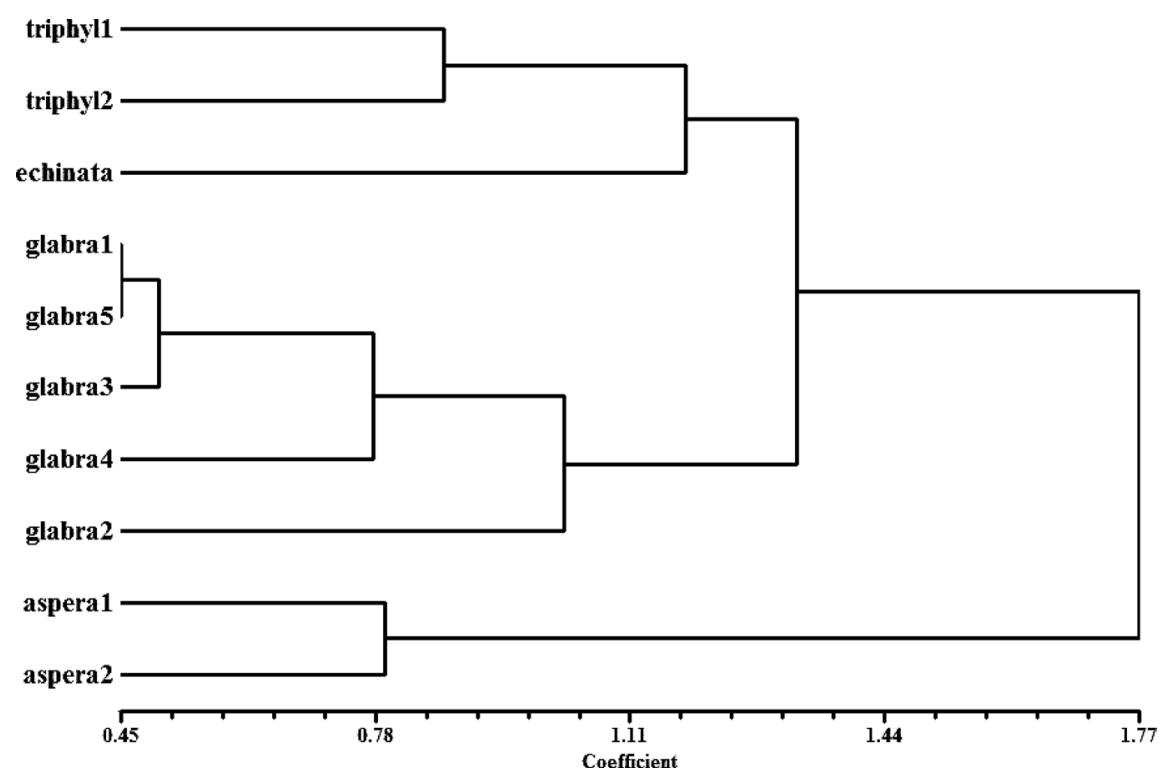

Fig. 1. UPGMA clustering of Glycyrrhiza species and populations based on quantitative morphological characters. (Species abbreviations $=$ glabra $1-5=$ G. glabra, Gorgan, Tehran, Ghazvin, Sorkhehesar and Hamedan populations respectively, asper $=G$. aspera Ghazvin and Gardaneh Koohin populations respectively, triphylla 1 and $2=G$. triphylla, Gorgan and Kordestan populations respectively, echinata $=$ G. echinata Rasht and Kordestan populations respectively.)

imens are deposited in TARI, IRAN and Herbarium of Shahid Beheshti University (HSBU).

For morphometric studies, 47 morphological characters (quantitative and qualitative) were used. The mean of quantitative characters were used while qualitative characters were coded as binary/multistate characters. Variables were standardized $($ mean $=0$, variance $=1$ ) for multivariate statistical analyses. In order to group the species having morphological similarities, cluster analysis using UPGMA (Unweighted Paired Group with Arithmetic Average) and WARD (Minimum Variance Spherical Clusters) as well as ordination based on principal component analysis (PCA) were performed (Chatfield and Collins 1995). The Euclidean distance/ squared Euclidean distance was used as dissimilarity coefficient in cluster analysis of morphological data. In order to determine the most variable morphological characters among the species/populations, factor analysis based on principal components analysis (PCA) was performed.

\section{Cytogenetic studies}

Young flower buds were collected from 10 randomly selected plants of each species/population and fixed in glacial acetic acid: ethanol $(1: 3)$ for $24 \mathrm{~h}$. Flower buds were washed and preserved in $70 \%$ ethanol at $4{ }^{\circ} \mathrm{C}$ until used (Sheidai et al. 2005). Cytological preparations used squash technique and $2 \%$ aceto-orcein as the stain.

Fifty to one hundred pollen mother cells (PMCs) were analysed for chiasma frequency and distribution at diakinesis and metaphase stages while 500 PMCs were analysed for chromosome segregation during the anaphase and telophase stages. Pollen satiability as a measure of fertility was determined by staining minimum 1000 pollen grains with $2 \%$ acetocarmine : 50\% glycerin $(1: 1)$ for about $1 / 2$ hr. Round. Complete pollens which were stained were taken as fertile, while incomplete, shrunken pollens with no stain were considered as infertile (Sheidai et al. 2005).

Analysis of variance (ANOVA) followed by the Least Significant Test (LSD) was performed among populations as well as different species studied to detect any significant difference in the rel- 
ative chiasma frequency and distribution as well as chromosomes association while, $\chi^{2}$ test was performed to detect a significant difference in meiotic abnormalities (Sheidai et al. 2005).

In order to test the fitness of different clustering trees to the original similarity of the species studied, cophenetic correlation was determined. SPSS ver. 9 (1998) and NTSYS ver. 2.02 (1998) were used for statistical analyses.

\section{Results and discussion}

\section{Morphometry}

ANOVA and LSD test of quantitative morphological characters showed significant difference in most of the characters among the populations of a species and among the species studied (data not shown), so it seemed at the beginning that the quantitative morphological characters may not be useful in taxonomy of the Glycyrrhiza species but clustering of the species by using only quantitative morphological characters (Fig. 1), showed distinctness of the species studied. The populations of each species were placed together in a single cluster separated from the other species. Clustering of the glycyrrhiza species based on all morphological characters produced similar grouping as quantitative morphological characters, separating the species in distinct groups therefore it seems that both quantitative and qualitative morphological characters may be used in taxonomy of the genus Glycyrrhiza. The most variable morphological characters identified by factor analysis i.e. the pod shape, ratio of length to width of leaflets; the number of leaflet pairs and shape of stipule may be useful in Glycyrrhiza species delimitation.

Dendrogram obtained by UPGMA clustering possesses the highest cophenetic correlation $(r=0.92)$ and the best fit to the original data, therefore the species relationships is discussed on this dendrogram. The populations of G. glabra form the first major cluster separated from the other species (Fig. 1). Two species of G. triphylla and G. echinata show similarity and comprise the second cluster, while populations of $G$. aspera form the third cluster. RAPD and RFLP analyses also have shown that G. glabra is closer to other Glycyrrhiza species than to G. echinata (Yamazaki et al. 1994). PCA ordination of the species based on morphological characters also supported the clustering result.

Factor analysis revealed that the first two PCA factors comprise about $70 \%$ of total variance. In the first factor with about $47 \%$ of total variation, characters like the ratio of length to with of pod, shape of leaflets, length of standard, length of gynecium and pubescence of calyx pos- 

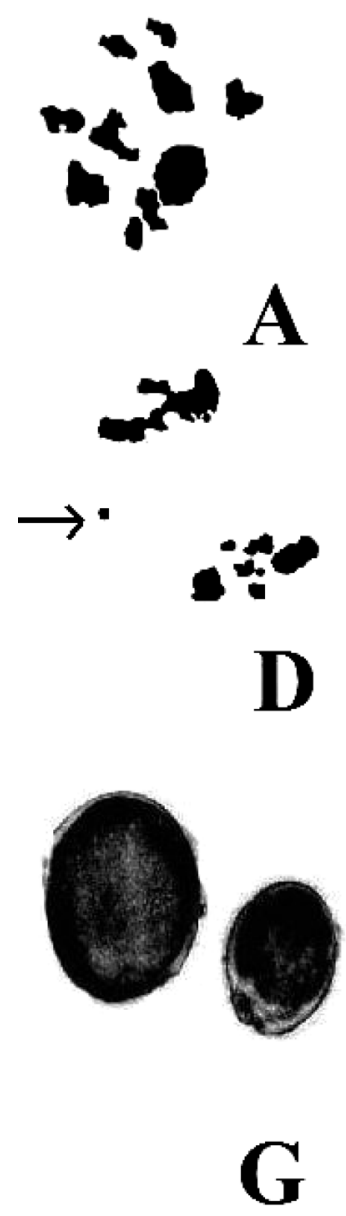

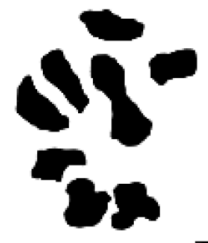

B

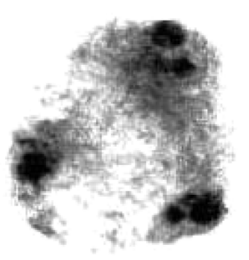

$\mathbf{E}$
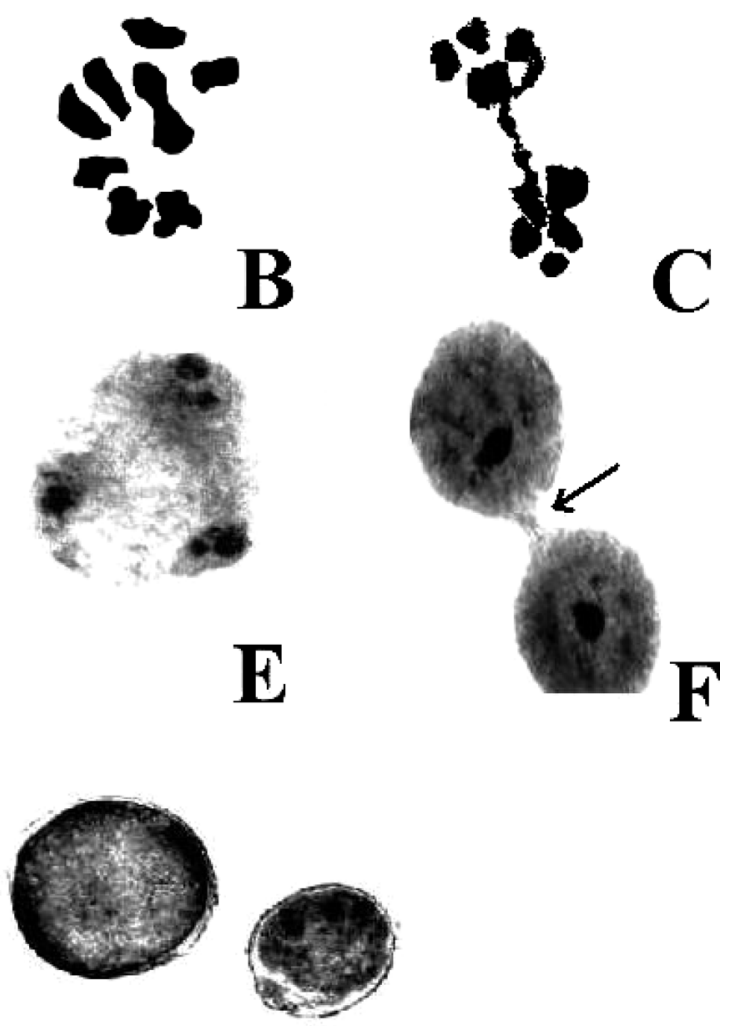

$\mathbf{H}$

Fig. 2. Representative meiocytes in Glycyrrhiza species studied. A and $\mathrm{B}=$ Meiocytes showing $n=8$ in $G$. aspera and $G$. triphylla respectively. $\mathrm{C}$ and $\mathrm{D}=$ Meiocytes showing anaphase stickiness and laggard chromosome (arrow) in G. echinata and G. glabra respectively. E=A tripolar cell in G. triphylla. $\mathrm{F}=$ Cytomictic channel (arrow) among neighboring meiocytes in G. glabra. $\mathrm{G}=$ Potential unreduced (bigger sized) pollen in G. triphylla. $\mathrm{H}=$ Potential unreduced (bigger sized) pollen in G. aspera. Scale bar $=10 \mu \mathrm{m}$.

sessed the highest positive correlation $(r=0.90)$.

In the second factor with about $14 \%$ of total variance, characters like the pod shape, ratio of length to width of leaflets, the number of leaflet pairs and shape of stipule possessed the highest positive correlation $(r=0.70)$. Morphological characters of the first PCA factor mainly separates $G$. aspera form the other species while variable morphological characters of the second PCA factor mainly separates G. glabra from the other Glycyrrhiza species studied.

\section{Cytogenetic studies}

Cytogenetical studies revealed the presence of $2 n=2 x=16$ chromosome number in all the species studied (Table 1, Fig. 2). The presence of $2 n=2 x=16$ chromosome number have been reported before in the species of G. aspera, G. glabra and G. echinata (Nafanailova 1989, Li and Wei 1992, Ghaffari and Chariat-Panahi 1985), but the chromosome number of G. triphylla is a new report. 


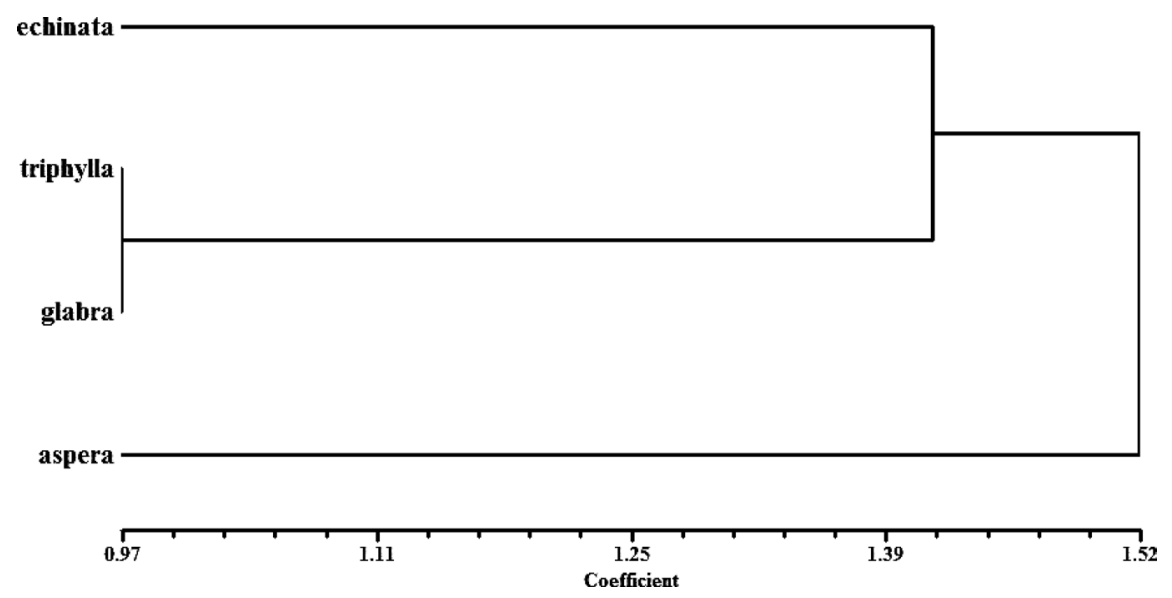

Fig. 3. UPGMA clustering of Glycyrrhiza species and populations based on cytogenetic data. Species abbreviations: echinata $=$ G. echinata, aspera $=$ G. aspera, triphyla $=$ G. triphylla and glabra $=$ G. glabra.

The chromosomes formed mostly bivalents in metaphase-I with a low amount of univalents. The highest value of total and terminal chiasmata occurred in G. echinata (13.53 and 12.31 respectively), while the highest value of intercalary chiasmata occurred in G. triphylla (2.35, Table 1).

Almost in all the species studied, laggard chromosomes and chromosomes stickiness were observed during anaphase-I and II (Table 1, Fig. 2). The sticky chromosomes occurred from early stages of prophase to the final stages of meiosis in most of the species studied. The number of chromosomes involved in stickiness varied from two to many forming a complete clumping of the chromosomes. The highest percentage of stickiness during anaphase-I and II occurred in G. aspera (6.10 and 6.66 respectively). The $\chi^{2}$ test showed that G. aspera differs significantly in the percentage of chromosome stickiness and laggard chromosomes from the other Glycyrrhiza species studied.

The occurrence of mostly bivalents in metaphase of meiosis I and almost normal chromosomes segregation during anaphase may be related to diploid nature of Glycyrrhiza species, however the occurrence of some amount of meiotic abnormalities including chromosome stickiness, cytomixis and the formation of unreduced gametes observed may lead to some degree of genetic variability in the next generation progenies. Genetic and environmental factors as well as genomic-environmental interaction have been considered as the reason for chromosome stickiness in different plant species (Nirmala and Rao 1996, Baptista-Giacomelli et al. 2000).

ANOVA test did not show significant difference for chromosome pairing and chiasma frequency among the species studied indicating that there has not been a significant change in the number of genes controlling chiasma formation and chromosome pairing during Glycyrrhiza species diversification. However, significant difference observed in the percentage of laggard chromosomes and chromosome stickiness among the species studied may partly indicate their genomic difference. Such meiotic irregularities may be the reason for the low amount of pollen sterility observed in Glycyrrhiza species (Table 1).

Multipolar cells, abnormal tetrads and abnormal pollen grains were observed in the species studied ranging from $0.81 \%$ in G. echinata to $8.47 \%$ in G. glabra (Table 1, Fig. 2). Pair-wise $\chi^{2}$ test performed showed a significant difference in the percentage of cells showing such meiotic abnormalities among the species studied.

The occurrence of large pollen grains (possibly $2 n$ pollen grains) was observed along with smaller (normal) pollen grains in all the species studied (Fig. 2). The mean diameter of normal (re- 
duced) pollen grains was $5.00 \mu \mathrm{m}$ while the mean diameter of unreduced pollen grains was $10.00 \mu \mathrm{m}$, comprising about $2 \%$ of total pollen grains in Glycyrrhiza species studied. T-test analysis revealed a significant difference $(p<0.001)$ for the size between the larger sized pollen grains and smaller sized pollen grains.

Multipolar cell formation has been reported to cause abnormal tetrad and pollen grain formation, aneuploidy condition and chromosome mosaics in different plant species, including derivatives of Aegilops $\times$ Triticum hybrids, amphiploids of Solanum hybrids, as well as different species of Bromus and pomegranate cultivars (Villeux 1985, Nirmala and Rao 1996, Sheidai and Nouroozi 2005, Sheidai et al. 2005). The spindle apparatus is normally bipolar and acts as a single unit, playing a crucial role in chromosome alignment during metaphase. Any distortion or breakage in the spindle may result in random sub-grouping. Different reasons have been suggested for the occurrence of spindle abnormalities including: duality of nucleus in foreign cytoplasm, environmental influence and disharmonious gene interaction (Nirmala and Rao 1996).

The presence of giant pollen grains has been used as an indication of the production of $2 n$ pollen (Vorsa and Bingham 1979). Multipolar cells may also lead to the formation of unreduced $(2 n)$ pollen grains (larger pollen grains) observed in Glycyrrhiza species studied. Unreduced gametes are known to produce individuals with higher ploidy level through a process known as sexual polyploidization (Villeux 1985), which has been considered as the major route to the formation of naturally occurring polyploids. Different cytological mechanisms are responsible for the production of $2 n$ gametes including anaphase failure, desynapsis, multipolar cell formation, etc. (Villeux 1985). To our knowledge this is the first report on the occurrence of multipolar cell and unreduced pollen grain formation in the genus Glycyrrhiza.

UPGMA clustering (Fig. 3) of the species based on cytogenetic data, showed close relationship between G. glabra and G. triphylla and also between G. echinata and G. aspera, supporting the morphological results. Therefore the present study shows the use of cytological and morphological in revealing the species relationship in the genus Glycyrrhiza, reporting the chromosome number of G. triphylla and the occurrence of unreduced pollen grains for the first time.

\section{References}

Barghi, N. and Siljak-Yakovlev, S. 1990. Karyological study in three species of Glycyrrhiza genus (G. glabra, G. lepidota and G. echinata). Caryologia 43: 223-234.

Baptista-Giacomelli, F. R., Pagliarini, M. S. and Almeida, J. L. 2000. Meiotic behavior in several Brazilian oat cultivars (Avena sativa L.). Cytologia 65: 371-378.

Chatfield, C. and Collins, A. J. 1995. Introduction to Multivariate Analysis. Chapman \& Hall, London.

Ghaffari, S. M. and Chariat-Panahi, M. S. 1985. Chromosome count of some angiosperms from Iran. Iranian J. Bot. 3: 67-73.

Krausse, R., Bielenberg, J., Blaschek, W. and Ullmann, U. 2004. In vitro anti-Helicobacter pylori activity of Extractum liquiritiae, glycyrrhizin and its metabolites. J. Antimicrob. Chemothera. 54: 243-246.

Li, X. Y. and Wei, L. J. 1992. The karyotype analyses of some new taxa of Glycyrrhiza. Advan. Plant Taxonomy North China 1: 101-107.

Nafanailova, I. I. 1989. Examination, caryologica comparativa Glycyrrhiza L. et Meristotropis Fisch. et Mey. Botanicheskie Materialy Gerbariia Instituta Botaniki Akademii Nauk Uzbekskoi SSR 2: 51-68.

Nirmala, A. and Rao, P. N. 1996. Genetics of chromosome numerical mosaism in higher plants. The nucleus 39: 151-175.

Rechinger, K. H. 1984. Papilionaceae. Flora Iranica. Vol. 151. Academische Druck-U. Verlaganstalt.

Sheidai, M. and Nouroozi, M. 2005. Cytological studies on some species of Bromus sect. Bromus. Bot. Lithua. 11: 141-150.

-, M., Khandan, M. and Nasre-Esfahani, Sh. 2005. Cytogenetical study of some Iranian pomegranate (Punica granatum L.) cultivars. Caryologia 58: 132-139.

Verma, S. and Nadkarni, R. S. 1985. Chromosome numbers and karyotypic studies in Glycyrrhiza. Curr. Sci. 54: 45-47.

Villeux, R. 1985. Diploid and polyploid gametes in Crop Plants: Mechanisms of formation and utilization in plant breeding. In: Janick, J. (ed.), Plant Breeding Review. Vol. 3. 442 p. AVI Publishing Co. Wesport, Connecticut.

Vorsa, N. and Bingham, E. 1979. Cytology of $2 n$ pollen formation in diploid alfalfa, Medicago sativa. Can. J. Genet. Cyto. 
21: 525-530.

Yamazaki, M., Sato, A., Shimomura, K., Saito, K. and Murakoshi, I. 1994. Genetic relationships among Glycyrrhiza plants determined by RAPD and RFLP analyses. Biol. Pharm. Bull. 17: 1529-1531. 